\title{
Reviewers for Mayo Clinic Proceedings (2012)
}

Many individuals have given generously of their time and expertise to review manuscripts for Mayo Clinic Proceedings this past year. We are grateful to each of these reviewers. Locations are provided for those who are not affiliated with Mayo Foundation.-Editor

John P. Abenstein

Freddy Abi-Samra

New Orleans, LA

David A. Ahlquist

Mark R. Albertini

Madison, WI

Thomas G. Allison

Ashwin Ananthakrishnan

Boston, MA

Nandan S. Anavekar

Dallas W. Anderson

Bethesda, MD

Stephen M. Ansell

David R. Andes

Madison, WI

Ross Arena

Albuquerque, NM

Aneel A. Ashrani

Samuel J. Asirvatham

Sumeet Asrani

Steven Babin

Laurel, MD

Navkaranbir S. Bajaj

Cleveland, $\mathrm{OH}$

Charles M. Balch

Baltimore, MD

David J. Ballard

Dallas, TX

Kevin M. Barrett

George B. Bartley

Philippe R. Bauer

Robert L. Beck Jr

Nashville, TN

Thomas J. Beckman

Gianni Bellomo

Foligno, Italy

David G. Benditt

Minneapolis, MN

Robert Bennett

Portland, OR

Keith H. Berge

Kathy Berra

Palo Alto, CA

Julie A. Bjoraker

Morris J. Blachman

Columbia, SC

Mel Blumenthal

Minneapolis, MN
Amy Bonomi

Columbus, $\mathrm{OH}$

Jeremy Bordeaux

Cleveland, $\mathrm{OH}$

Aleta A. Borrud

J. Michael Bostwick

Juan M. Bowen

Ronald S. Brookmeyer

Los Angeles, CA

Robert D. Brown Jr

Alison J. Bruce

Martha L. Bruce

New York, NY

Alan H. Bryce

Christopher M. Burkle

Michael Calderwood

Boston, MA

Jeffrey Callen

Louisville, KY

Andrew D. Calvin

Elizabeth J. Campagna

Aurora, CO

Paul E. Carns

Gregory D. Cascino

Petra M. Casey

Renee E. Caswell

Alanna M. Chamberlain

William P. Cheshire

Christine Chhakchhuak Chattanooga, TN

Yung-Wei (Willi) Chi

Sacramento, CA

Vineet Chopra

Ann Arbor, MI

Lisa Christopher-Stine Baltimore, MD

Patricia Chute Old Westbury, NY

Bart L. Clarke

Nerissa M. Collins

S. Charles Collins

Nneka I. Comfere

Denis A. Cortese

Phoenix, AZ

Douglas B. Coursin

Madison, WI

Gregory Crosby

Boston, MA
Julien Crouzet Besancon, France

Mary Eliot Crowley

Philip Cryer St Louis, MO

Michael W. Cullen

Deborah J. Culley

Boston, MA

Burke A. Cunha

Mineola, NY

David R. Danielson

Mark Denis P. Davis

John de Figueiredo Cheshire, CT

Francoise Delehaye Bron, France

John J. Dillon

David Dingli

Roxana S. Dronca

Liselotte (Lotte) N. Dyrbye

Walter Dzik

Boston, MA

Conrad P. Earnest Bath, United Kingdom

Jeanette E. Eckel Passow

Peter Ehrlich

Ann Arbor, MI

Benjamin H. Eidelman

Mackram F. Eleid

Richard M. Elias

Muhamad Y. Elrashidi

Mark J. Enzler

Lori A. Erickson

Lynn L. Estes

N. A. Mark Estes

Boston, MA

Julie A. Fairman Philadelphia, PA

Wassim H. Fares Chapel Hill, NC

Philip R. Fischer

Jerome L. Fleg

Bethesda, MD

Gerald Fletcher

Christy Foreman

Silver Spring, MD
Daniel E. Forman

Boston, MA

Jean C. Fox

Andrew Franks

New York, NY

Robert L. Frye

Jimmy R. Fulgham

Cliff Fullerton

Dallas, TX

W. Bruce Fye

Lance Gable

Detroit, MI

Susan Galandiuk

Louisville, KY

Harsha Ganga

St Paul, MN

Beng Gardlund

Stockholm, Sweden

Joan Manel Gasent Blesa

Dénia, Spain

Thomas C. Gerber

Dale Gerding

Hines, IL

Richard Gilroy

Kansas City, KS

Richard Glassock Laguna Niguel, CA

Kashish Goel

Detroit, MI

Richard M. Goldberg

Columbus, $\mathrm{OH}$

Corey Goldman

New Orleans, LA

William B. Grant

San Francisco, CA

Roy A. Greengrass

Andrew C. Greenlund

Thomas M. Habermann

John B. Hagan

Albert G. Hakaim

Matthew R. Hall

Stephen C. Hammill

James D. Hannon

Gregory J. Hanson

Iana H. Haralambieva

Robert E. Harbaugh

Hershey, PA

Robert P. Hartman 
Robert P. Heaney

Omaha, NE

Michael G. Heckman

John E. Heffner

Portland, OR

Raymond L. Heilman

John A. Heit

Walter C. Hellinger

David C. Herman

Greenville, NC

Roland Herrero Lyon, France

Laurence J. Hirsch Franklin Lakes, NJ

Sara E. Hocker

Margaret L. Hoffman-Terry Allentown, PA

Thomas F. Hogan Morgantown, WV

Michael F. Holick Boston, MA

Jennifer Hsu Sioux Falls, SD

Jian Huang

Fresno, CA

Richard D. Hurt

John Huston III

Vivek N. Iyer

Steven J. Jacobsen

Pasadena, CA

Allan S. Jaffe

James W. Jakub

Christopher J. Jankowski

Stephen G. Jenkins New York, NY

Rebecca L. Johnson

Lanetta Jordan Cooper City, FL

Michael J. Joyner

Mary L. Jurisson

Peter Kam Sydney, Australia

Sunanda V. Kane

Hyun Gu Kang

Pomona, CA

Dean G. Karalis

Philadelphia, PA

Mary J. Kasten

Lia C. Kaufman

Judith S. Kaur

Mark T. Keegan

A. Scott Keller

David L. Keller

Torrance, CA

Cassie C. Kennedy
James Kennedy

Toronto, Ontario

Sahil Khanna

Marjorie L. King

West Haverstraw, NY

Michael Kleerekoper

Ypsilanti, MI

Manish Kohli

David Korones Rochester, NY

Thomas E. Kottke Minneapolis, MN

Lois E. Krahn

Megan L. Krause

Daniel F. Kripke La Jolla, CA

Walter Kukull Seattle, WA

Amit Kumar Worcester, MA

Young Kwok Baltimore, MD

Loralie J. Langman

Carl J. Lavie New Orleans, LA

Thierry H. LeJemtel New Orleans, LA

Duck-chul Lee Ames, IA

John Hyung-il Lee New Orleans, LA

Linda S. Lee Boston, MA

Roberto Leone Verona, Italy

Kit Leung Montreal, Quebec

James T. C. Li

Mark Liebow

Lionel Lim Derby, CT

Wan-Yu Lin Taichung, Taiwan

Yi Lin

Noralane M. Lindor

Benjamin A. Lipsky Seattle, WA

Scott C. Litin

Conor G. Loftus

Ben Lomaestro

Albany, NY

Magnus Londahl Lund, Sweden

Francisco Lopez-Jimenez Phillip A. Low
Ingrid E. Lundberg Stockholm, Sweden

Larry B. Lundy

Mark K. Lyons

Daniel E. Maddox

Melinda Maggard Los Angeles, CA

Antonis S. Manolis Athens, Greece

Roberto Marchioli Santa Maria Imbaro, Italy

Scott Marsal Portland, OR

Eric L. Matteson

Karen F. Mauck

George Mayzell Memphis, TN

Timothy H. McCalmont San Francisco, CA

James T. McCarthy

Timothy McCashland Omaha, NE

Peter A. McCullough Novi, MI

Cliff McDonald Atlanta, GA

Michael D. McGoon

Paul M. McKie

Christopher J. McLeod

Robert R. McWilliams

L. Joseph Melton III

James F. Meschia

Franz H. Messerli New York, NY

Hector I. Michelena

Richard V. Milani New Orleans, LA

Loren G. Miller Torrance, CA

Wayne L. Miller

Martin M. Miner Providence, RI

Kevin G. Moder

David N. Mohr

Jonathan Moss Chicago, IL

Timothy J. Moynihan

Paul S. Mueller

J. (Joseph) Brent Muhlestein Murray, UT

Manpreet S. Mundi Joseph G. Murphy Joshua B. Murphy Joseph A. Murray Philip R. Muskin New York, NY
James M. Naessens

Bradly J. Narr

Udayakumar Navaneethan Cleveland, $\mathrm{OH}$

Robert E. Nesse La Crosse, WI

Dumbor L. Ngaage Cottingham, United Kingdom

Vuyisile T. Nkomo

James G. O'Brien

Louisville, KY

James H. O'Keefe Kansas City, MO

Michael F. O'Rourke Sydney, Australia

Jae K. Oh

Robert Orenstein

Antigone Oreopoulos Edmonton, Alberta

Clark C. Otley

Animesh Pardanani

Sung Keun Park

Seoul, Korea

Jeffrey J. Pasternak

Ashok M. Patel

Maitray D. Patel

Robin Patel

Mrinal S. Patnaik

Nancy J. Petersen Houston, TX

Michael C. Peterson Provo, UT

Sorin V. Pislaru

Didier Pittet Geneva, Switzerland

Gregory A. Poland

Nicholas Poolos Seattle, WA

Michael K. Popkin Minneapolis, MN

Rajiv K. Pruthi

Qi Qian

Alejandro A. Rabinstein S. Vincent Rajkumar Matthew A. Rank Keith G. Rasmussen Michael Ready Temple, TX John J. P. Reilly New Orleans, LA Elliott Richelson Richard J. Rodeheffer Yvonne Romero Steven H. Rose 
Warren Rose

Madison, WI

Heather Rosecrans Washington, DC

Edward C. Rosenow

John C. Rowlingson

Charlottesville, Virginia

Andrew D. Rule

James R. Rundell

William Sanchez

Nicole P. Sandhu

Paula J. Santrach

William J. Savage

Baltimore, MD

Borja Sañudo Seville, Spain

Hartzell V. Schaff

Delia Scholes

Seattle, WA

Georgene Schroeder Rochester, MN

Henry J. Schultz

Gary L. Schwartz

Marin L. Schweizer Iowa City, IA

Benjamin Scirica Boston, MA

Raymond C. S. Seet Singapore, Singapore

F. John Service

Pankaj Shah

Alan Shindel

Sacramento, CA

Marina G. Silveira

Marc A. Silver

Oak Lawn, IL
Marc D. Silverstein

Mashpee, MA

Joseph I. Sirven

Susan L. Slager

Andrzej T. Slominski

Memphis, TN

Steven A. Smith

Jeffrey Soar

Toowoomba, Australia

Benjamin M. Solomon

Ulrich Specks

Robert J. Spinner

Arjun Srinivasan

Atlanta, GA

Jennifer St. Sauver

Anthony A. Stans

Stavros Stavrakis Oklahoma City, OK

Theodore A. Stern

Boston, MA

William G. Stevenson

Boston, MA

William Stewart

Cleveland, $\mathrm{OH}$

Craig S. Stump

Columbia, MO

Shajan Sugandha Birmingham, AL

Xuemei Sui

Columbia, SC

Christina M. Surawicz Seattle, WA

Jayant A. Talwalkar

Paul V. Targonski

William Tatum

Ayalew Tefferi
Zelalem Temesgen

Tom D. Thacher

Uma Thanarajasingam

Wendy E. Theobald Madison, WI

Randal J. Thomas

Jon C. Tilburt

Farris K. Timimi

Rochelle B. Torgerson

Hung-Fu Tseng Pasadena, CA

Joseph R. Tucci Providence, RI

Gerard M. Turino New York, NY

Bert B. Vargas

Robert Vassallo

Adrian Vella

Hector O. Ventura

New Orleans, LA

Anil Verma

New Orleans, LA

Robert A. Vogel

Baltimore, MD

Dietlind Wahner-Roedler

Hongsheng Wang

Nanjing, China

Zhen Wang

David O. Warner

Mark A. Warner

Mary Ellen Warner

D. Watanabe

Nagakute, Japan

Pamela (Pam) J. Weagraff

Norfolk, MA

Catherine R. Weiler
Ruth S. Weinstock Syracuse, NY

Howard Weitz Philadelphia, PA

Gail A. Welsh

David A. Wetter

Robert E. Wharen Jr

Roger D. White

Kevin J. Whitford

Carilyn N. Wieland

Mark L. Wieland

Aimee M. Wilkin

Winston Salem, NC

Floyd B. Willis

Christopher M. Wittich

Alexandra P. Wolanskyj

Bruce Wolffenbuttel

Groningen, Netherlands

Peter C. Wollan

Rochester, MN

Joseph P. Wood

Keh-Gong Wu

Taipei, Taiwan

Waldemar E. Wysokinski

Barbara P. Yawn

Rochester, MN

Roy A. Yawn

Rochester, MN

Paul R. Young

Steven Zeliadt

Seattle, WA

Richard S. Zimmerman

Christina K. Zorn 